\title{
Articular cartilage: Lamellar-repulsive lubrication of natural joints
}

\author{
Zbigniew A Figaszewski* \\ Institute of Chemistry, University of Bialystok, Al. Pilsudskiego 11/4, 15-443 Bialystok, Poland
}

\begin{abstract}
This monograph attempts to explain a new joint lubrication mechanism with surface active phospholipids as a lubricant. It provides studies of the principles of cartilage (smart material) of biological micro-and nanotribology.
\end{abstract}

\section{Book review}

This monograph presents a comprehensive approach to articular surface physics and biochemistry with an emphasis laid on the lamellarrepulsive mechanism of lubrication. Frictionless mechanical interaction of the charged positively, charged negatively and neutral surfaces (cartilage/cartilage; $(+/+) ;(-/-)$; neutral $( \pm) /( \pm)$ ) is strictly educational for biomechanical engineering.

The first part of the book reviews the results of structure, composition and function of the cartilage phospholipid (PL) bilayers as a potential solid lubricant. The authors' original contributions, focused on interfacial energy of PL bilayers with the emphasis lay on amphoteric character of fixed charged cartilage surfaces, are quite original.

The second part of the book deals with the friction vs wettability and presents the condition of cartilage surface correlated with surface wettability (contact angle). A change in natural cartilage surface energy leads to conformational changes in the surface of bovine patella from bilayer (super hydrophilic, $\sim 0^{\circ}$ contact angle wettability) to monolayer (hydrophobic $104^{\circ}$ contact angle wettability) during the process of airdrying. The biological tissue of the cartilage in its natural condition, transforming from the hydrophilic to hydrophobic condition, can be named a "smart material".

The third part of the book consists of chapters covering regeneration, resurfacing and restoration of the degraded surfaces, as well as the challenges and possibilities of achieving a biological knee repair for human patients.

All these experimental facts including surface energy, wettability, friction measurements were performed by the author when studying the boundary-layered friction supporting the lamellar-repulsive mechanism of lubrication of natural joints.

The biotribological model described in the monograph provides a new approach to the lubrication of natural joints and it certainly deserves to be learned by students. In the opinion of the Reviewer, investigations of the lamellar-electrostatic lubrication mechanism are very useful and innovative and deserve to be at the library.

This monograph is intended for advanced undergraduate and graduate students and researches active in the area of biomechanics and with biological systems, and engineers interested and involved in the sciences of nanomaterials (Glimpse of Book in Figure 1).

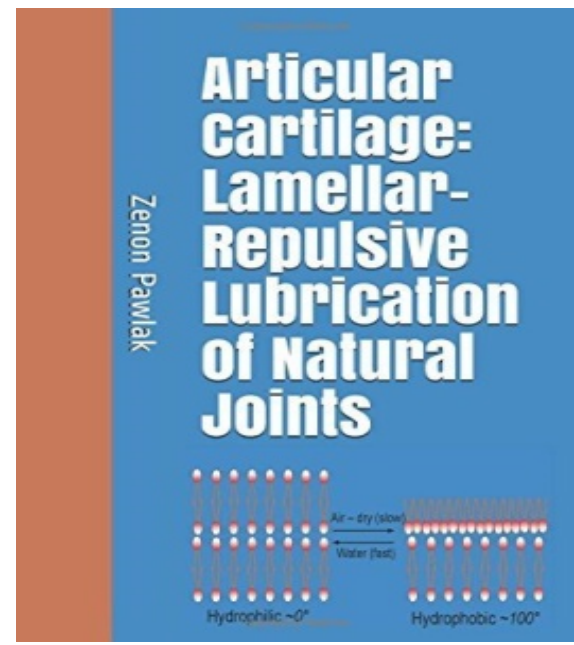

\section{Contents}

1.0 Introduction

2.0 The structure, composition and function of cartilage

2.1 Synovial fluid

2.2 Phospholipid bilayers as a potential solid lubricant

2.3 Phospholipids

2.4 Cartilage and antiphospholipid antibody syndrome

${ }^{*}$ Correspondence to: Zbigniew A Figaszewski, Institute of Chemistry, University of Bialystok, Al. Pilsudskiego 11/4, 15-443 Bialystok, Poland, E-mail: figaszew@uwb.edu.pl

Key words: articular cartilage, natural lubrication, lamellar-repulsive mechanism of lubrication of natural joints

Received: June 18, 2018; Accepted: June 22, 2018; Published: June 30, 2018 
3.0 Surface cartilage characteristics

3.1 Interfacial energy of PL bilayers

3.2 Cartilage surface wettability vs. $\mathrm{pH}$

3.3 Friction vs. wettability of cartilage surface

3.4 Amphoteric cartilage and its consequences

3.5 Cartilage surface charge density vs. $\mathrm{pH}$

4.0 Porosity of lubricated cartilage surface

5.0 The boundary and boundary-layered lubrication

6.0 Lamellar-repulsive mechanism of low friction in Nature

7.0 Resurfacing cartilage surface
8.0 Articular cartilage restoration and regeneration

9.0 Summary and outlook

About the Author

Bibliography

64

Index

\section{Reference}

85

89

1. Pawlak, Zenon (2018) Articular Cartilage: Lamellar-Repulsive Lubrication of Natural Joints, Kindle Direct Publishing, 171pp. Print-book: https://www.amazon.com/dp/B07B42P1JY, e- book: https://www.amazon.com/dp/1976760283.

Copyright: $(2018$ Figaszewski ZA. This is an open-access article distributed under the terms of the Creative Commons Attribution License, which permits unrestricted use, distribution, and reproduction in any medium, provided the original author and source are credited. 